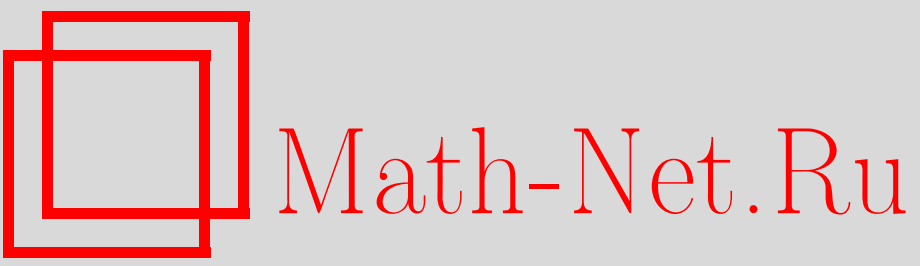

А. С. Холево, М. Е. Широков, Проблема аддитивности для квантовых каналов с ограничениями, УМH, 2004, том 59, выпуск 2, 195-196

DOI: https://doi.org/10.4213/rm729

Использование Общероссийского математического портала Math-Net.Ru подразумевает, что вы прочитали и согласны с пользовательским соглашением

http://www.mathnet.ru/rus/agreement

Параметры загрузки:

IP: 54.198 .67 .100

26 апреля 2023 г., 13:07:08 


\title{
ПРОБЛЕМА АДДИТИВНОСТИ ДЛЯ КВАНТОВЫХ КАНАЛОВ С ОГРАНИЧЕНИЯМИ
}

\author{
А. С. Холево, М. Е. ШиРоков
}

Одной из центральных проблем квантовой теории информации [1] является гипотеза об аддитивности классической пропускной способности квантовых каналов связи [2]-[4]. Недавно П. Шор показал [5], что выполнимость этой гипотезы для всех квантовых каналов эквивалентна целому ряду свойств (супер)аддитивности других важных характеристик таких, как минимальная выходная энтропия и мера сцепленности (entanglement of formation). В настоящей работе вводится новое свойство сильной аддитивности (аддитивности для каналов с ограничениями на входе), дается ряд его равносильных формулировок, устанавливается его выполнимость для некоторых нетривиальных классов каналов и отмечается, что выполнимость гипотезы аддитивности для всех каналов влечет сильную аддитивность.

Пусть $\mathscr{H}, \mathscr{H}^{\prime}$ - конечномерные унитарные пространства. Квантовым каналом называется линейное вполне положительное сохраняющее след отображение $\Phi: \mathfrak{B}(\mathscr{H}) \mapsto \mathfrak{B}\left(\mathscr{H}^{\prime}\right)$, где $\mathfrak{B}(\mathscr{H})$ - алгебра всех операторов в $\mathscr{H}$. В частности, $\Phi$ порождает аффинное отображение выпуклого множества $\mathfrak{S}(\mathscr{H})$ состояний (операторов плотности) на пространстве $\mathscr{H}$ в множество $\mathfrak{S}\left(\mathscr{H}^{\prime}\right)$ состояний на пространстве $\mathscr{H}^{\prime}[1]$.

Квантовый аналог теоремы Шеннона, установленный в [4], дает следующее выражение для классической пропускной способности:

$$
C(\Phi)=\lim _{n \rightarrow \infty} n^{-1} \bar{C}\left(\Phi^{\otimes n}\right),
$$

где $\Phi^{\otimes n}=\Phi \otimes \cdots \otimes \Phi-n$-я тензорная степень канала $\Phi$,

$$
\bar{C}(\Phi)=\max _{\left\{\pi_{i}, \rho_{i}\right\}}\left\{H\left(\sum_{i} \pi_{i} \Phi\left(\rho_{i}\right)\right)-\sum_{i} \pi_{i} H\left(\Phi\left(\rho_{i}\right)\right)\right\} .
$$

Здесь $H(\rho)=-\operatorname{Tr} \rho \log \rho$ - энтропия фон Неймана, а максимум берется по произвольньм входным ансамблям $\left\{\pi_{i}, \rho_{i}\right\}$, представляющим собой конечньй набор состояний $\left\{\rho_{i}\right\} \subset \mathfrak{S}(\mathscr{H})$ с соответствующими вероятностями $\left\{\pi_{i}\right\}$. Гипотеза аддитивности состоит в том, что

$$
\bar{C}(\Phi \otimes \Psi)=\bar{C}(\Phi)+\bar{C}(\Psi)
$$

где $\Phi \otimes \Psi$ - тензорное произведение каналов $\Phi, \Psi$. Ее выполнимость для фиксированного канала $\Phi$ и произвольного $\Psi$ влечет, в частности, равенство $\bar{C}(\Phi)=C(\Phi)$. Обзор относящихся сюда результатов см. в [3], [5].

Обозначая $\chi_{\Phi}\left(\left\{\pi_{i}, \rho_{i}\right\}\right)$ выражение в фигурных скобках в $(1)$, введем функцию

$$
\chi_{\Phi}(\rho)=\max _{\rho_{\mathrm{av}}=\rho} \chi_{\Phi}\left(\left\{\pi_{i}, \rho_{i}\right\}\right)=H(\Phi(\rho))-\widehat{H}_{\Phi}(\rho),
$$

где $\rho_{\text {av }}=\sum_{i} \pi_{i} \rho_{i}-$ среднее ансамбля $\left\{\pi_{i}, \rho_{i}\right\}, \widehat{H}_{\Phi}(\rho)=\min _{\rho_{\mathrm{av}}}=\rho \sum_{i} \pi_{i} H\left(\Phi\left(\rho_{i}\right)\right)$ - выпуклое замькание [6] выходной энтропии канала $H(\Phi(\rho))$. Функция $\chi_{\Phi}(\rho)$, как и $H(\Phi(\rho))$, является вогнутой непрерывной функцией на $\mathfrak{S}(\mathscr{H})$. Введем также функцию

$$
\nu_{H}(\Phi, A)=\min _{\rho \in \mathfrak{S}(\mathscr{H})}[H(\Phi(\rho))+\operatorname{Tr} A \rho]
$$

которая является сопряженной к минималной выходной энтропии [6].

Пусть $\mathscr{A} \subseteq \mathfrak{S}(\mathscr{H})$ - некоторое замкнутое подмножество состояний на входе канала. Рассмотрим величину

$$
\bar{C}(\Phi, \mathscr{A})=\max _{\rho \in \mathscr{A}} \chi_{\Phi}(\rho)=\max _{\rho_{\mathrm{av}} \in \mathscr{A}} \chi_{\Phi}\left(\left\{\pi_{i}, \rho_{i}\right\}\right) .
$$

В приложениях $\mathscr{A}$ обычно определяется линейным неравенством $\operatorname{Tr} A \rho \leqslant \alpha$, где $A$ - некоторый положительньй оператор (например, энергия), $\alpha \geqslant 0$.

Работа выполнена при частичной поддержке грантов НШ 1758.2003.1 и ИНТАС 00-738. 
Рассмотрим два канала $\Phi: \mathfrak{S}(\mathscr{H}) \mapsto \mathfrak{S}\left(\mathscr{H}^{\prime}\right)$ и $\Psi: \mathfrak{S}(\mathscr{K}) \mapsto \mathfrak{S}\left(\mathscr{K}^{\prime}\right)$ с ограничениями, определяемыми множествами $\mathscr{A} \subseteq \mathfrak{S}(\mathscr{H})$ и $\mathscr{B} \subseteq \mathfrak{S}(\mathscr{K})$ соответственно. Для канала $\Phi \otimes \Psi$ естественно ввести "наследственное" ограничение, определяемое включениями $\omega_{\mathrm{av}}^{\Phi}=\operatorname{Tr}_{\mathscr{K}} \omega_{\mathrm{av}} \in \mathscr{A}$ и $\omega_{\mathrm{av}}^{\Psi}=\operatorname{Tr}_{\mathscr{H}} \omega_{\mathrm{av}} \in \mathscr{B}$, где $\omega_{\mathrm{av}}-$ среднее входного ансамбля $\left\{\mu_{i}, \omega_{i}\right\}$. Соответствующее подмножество в $\mathfrak{S}(\mathscr{H} \otimes \mathscr{K})$ обозначим $\mathscr{A} \otimes \mathscr{B}$. Гипотеза аддитивности для каналов с ограничениями состоит в том, что

$$
\bar{C}(\Phi \otimes \Psi ; \mathscr{A} \otimes \mathscr{B})=\bar{C}(\Phi ; \mathscr{A})+\bar{C}(\Psi ; \mathscr{B}) .
$$

Теорема 1. Пусть $\Phi$ и - произвольные каналы. Следующие свойства равносиль$H b l$ :

(i) равенство (6) имеет место для произвольных замкнутых подмножеств $\mathscr{A}$ и $\mathscr{B}$;

(ii) равенство (6) имеет место для подмножеств $\mathscr{A}$ и $\mathscr{B}$, определяемых соответственно неравенствами $\operatorname{Tr} A \rho_{\mathrm{av}} \leqslant \alpha u \operatorname{Tr} B \sigma_{\mathrm{av}} \leqslant \beta$ при произвольных $A, \alpha, B, \beta$;

(iii) $\chi_{\Phi \otimes \Psi}(\omega) \leqslant \chi_{\Phi}\left(\omega^{\Phi}\right)+\chi_{\Psi}\left(\omega^{\Psi}\right) \quad \forall \omega \in \mathfrak{S}(\mathscr{H} \otimes \mathscr{K})$;

(iv) $\widehat{H}_{\Phi \otimes \Psi}(\omega) \geqslant \widehat{H}_{\Phi}\left(\omega^{\Phi}\right)+\widehat{H}_{\Psi}\left(\omega^{\Psi}\right) \quad \forall \omega \in \mathfrak{S}(\mathscr{H} \otimes \mathscr{K})$;

(v) $\nu_{H}(\Phi \otimes \Psi, A \otimes I+I \otimes B)=\nu_{H}(\Phi, A)+\nu_{H}(\Psi, B) \forall A \in \mathfrak{B}_{+}(\mathscr{H}), \forall B \in \mathfrak{B}_{+}(\mathscr{K})$.

Если выполняется одно из этих эквивалентных свойств, то будем говорить, что для каналов $\Phi$ и $\Psi$ имеет место сильная аддитивность.

Рассмотрим канал $\Phi: \mathfrak{S}(\mathscr{H}) \mapsto \mathfrak{S}\left(\mathscr{H}^{\prime}\right)$, и пусть $E \in \mathfrak{B}(\mathscr{H}), 0 \leqslant E \leqslant I$. Пусть $q \in[0 ; 1]$ и $d \in \mathbb{N}=\{1,2, \ldots\}$. Расширение Шора $\widehat{\Phi}(E, q, d)$ с вероятностью $1-q$ действует как канал $\Phi$, а с вероятностью $q$ производит измерение в $\mathscr{H}$ с двумя исходами $\{0,1\}$, описываемое разложением единицы $\{I-E, E\}$. Если исход измерения 1 , то на выход посылаются $\log d$ бит классической информации, в противном случае - сигнал неудачи. Точное определение см. в [7], [5]. Интересна асимптотика, когда $q$ стремится к нулю, а $d$ - к бесконечности, причем отношение $q \log d=\lambda$ остается постоянньм. Тогда $\widehat{\Phi}$ с высокой вероятностью действует на входные состояния $\rho$ как $\Phi$, изредка посылая болшшое количество информации, пропорциональное величине $\operatorname{Tr} \rho E$. Это объясняет связь между пропускной способностью расширения $\widehat{\Phi}$ и канала $\Phi$ с линейным ограничением на входе.

TеOPEMA 2. Пусть $\Phi: \mathfrak{S}(\mathscr{H}) \mapsto \mathfrak{S}\left(\mathscr{H}^{\prime}\right)$ u $\Psi: \mathfrak{S}(\mathscr{K}) \mapsto \mathfrak{S}\left(\mathscr{K}^{\prime}\right)-$ произвольные каналь с фиксированным ограничением $\mathscr{B}$ на входе второго канала. Следующие утверждения эквивалентны:

(i) аддитивность (6) имеет место для канала $\Phi$ с произвольным замкнутым ограничением на входе и канала $\Psi$ с ограничением $\mathscr{B} ;$

(ii) свойство аддитивности выполняется асимптотически при $d \rightarrow \infty$ для последовательности каналов $\{\widehat{\Phi}(E, \lambda / \log d, d)\}_{d \in \mathbb{N}}$ с произвольным оператором $0 \leqslant E \leqslant I$ и произвольным $\lambda>0$ и канала $\Psi$ с ограничением $\mathscr{B}$.

Применяя эту теорему дважды, получаем

СлЕДСТВИЕ. Выполнимость гипотезы аддитивности (2) для всех каналов влечет свойство сильной аддитивности (6).

\section{СПИСОК ЛИТЕРАТУРЫ}

[1] А.С. Холево. Введение в квантовую теорию информации. М.: МЦНМО, 2002. [2] Г. Г. Амосов, Р. Ф. Вернер, А. С. Холево // Проблемы передачи информации. 2000. Т. 36. № 4. C. 25-34. [3] A.S. Holevo. http://www.imaph.nat.tu-bs.de/qi/problems/problems.html. [4] А. С. Холево // УМН. 1998. Т. 53. №6. С. 193-230. [5] P. Shor. Arxiv e-print quant-ph $/ 0305035,2003$. [6] А.Д. Иоффре, В.М. Тихомиров. Теория экстремальных задач. М.: Наука, 1974. [7] A.S. Holevo, M. E. Shirokov. Arxiv e-print quant-ph/0306196, 2003 . 